\title{
Prevalence and determinants of overweight and obesity among school-aged children and adolescents
}

\author{
Guy Ikambo Wanghi ${ }^{1}$, Leslie Lytle ${ }^{2}$, Zakayi Pius Kabututu ${ }^{3}$, Augustin Rudahaba Buhendwa ${ }^{4}$, Kiswaya \\ Ernest Sumaili $^{5}$ \\ ${ }^{1,5}$ University of Kinshasa Faculty of Medicine, Unit of Physiology, Department of Basic Sciences, RD Congo \\ ${ }^{2}$ University of North Carolina at Chapel Hill, Department of Health Behavior, Gillings School of Global Public Health, USA \\ ${ }^{3}$ University of Kinshasa Faculty of Medicine, Unit of Molecular Biology, Department of Basic sciences, RD Congo \\ ${ }^{4}$ University of Kinshasa Faculty of Medicine, Department of Physical Medicine and Rehabilitation, RD Congo \\ ${ }^{5}$ University of Kinshasa Faculty of Medicine, Renal Unit, Department of Internal Medicine, RD Congo
}

\section{Article Info}

Article history:

Received Apr 7, 2020

Revised Jul 30, 2020

Accepted Sep 11, 2020

\section{Keywords:}

Age

Gender

Body fat

Physical activity

Obesity

Overweight

\begin{abstract}
Obesity is a significant public health crisis affecting millions of children globally. The objective of this study was to identify the prevalence and associated factors of overweight/obesity among school children in Kinshasa, Democratic Republic of Congo (DRC). This was a descriptive crosssectional study among school children and adolescents $(n=1442)$ from Kinshasa selected using multistage sampling method. A structured questionnaire was used to collect behavioral data. The WHO AnthroPlus was used to calculate BMI (body mass index). SPSS version 21 was used for data analysis. Potential covariates were examined using chi-square tests followed by multivariate logistic regression analyzes The study found that out of 1442 students, $72 \%$ of the sample was at a healthy weight, $15 \%$ were underweight and nearly $13 \%$ were overweight or obese. The prevalence of overweight and obesity was higher in girls as compared with boys. The results of multivariate logistic regressions showed that the gender of children, category of age, percent body fat, eating fruits and vegetables, and physical activity levels were significantly associated with childhood overweight/obesity. One in eight children and adolescents (12.8\%) aged 6 to 18 years in Kinshasa going to primary and secondary schools were either overweight or obese.
\end{abstract}

This is an open access article under the CC BY-SA license.

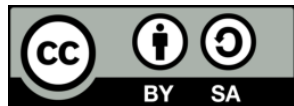

Corresponding Author:

Guy Ikambo Wanghi,

University of Kinshasa Faculty of Medicine,

Unit of Physiology,

Department of Basic sciences, RD Congo.

Email: guy.wanghi@unikin.ac.cd

\section{INTRODUCTION}

Mortality caused by overweight and obesity is growing worldwide, there are currently nearly 155 million overweight or obese children; it is known that the fifth risk of death in the world has become overweight and obesity [1]. School-aged children in sub-Saharan Africa have a growing incidence of overweight and obesity [2-5]. The prevalence of childhood overweight and obesity in Africa in 2010 was $8.5 \%$, and is expected to rise to $12.7 \%$ by 2020 , a relative increase of $49 \%$ [6]. Several authors believe that increased urbanization, industrialization, trade liberalization and economic growth are at the root of the current increasing prevalence of overweight in sub-Saharan Africa. These authors qualify these societal changes as the nutritional transition [7,8]. All of these changes have resulted in reduced physical 
activity and increased consumption of energy-dense foods [7, 8]. Although obesity-related illnesses mainly manifest in adulthood, the risks associated with the disease conditions generally originate in childhood [9]. Overweight and obese children are more likely to grow into overweight and obese adults with a higher chance of developing non communicable diseases like diabetes and cardiovascular disease [4, 9].

Kinshasa is the administrative and political capital of the DRC with a mixed population made of inhabitants from different provincial origins and socioeconomic backgrounds. The population in Kinshasa was estimated at 10.65 million in 2013 by the United Nations [10]. The rapid growth in population in Kinshasa that occurred in the last century brought with it increasing social inequity and poorer nutrition, hygiene and living conditions. These social conditions may be related to the increased risk of childhood obesity experienced.

However, in the DRC, studies on overweight and obesity of school aged children and adolescents are rare. A report by Buhendwa and others in 2017 on nutritional status, height, weight and BMI centiles of school-aged children and adolescents in the city of Kinshasa showed the prevalence of overweight around $8.6 \%$ and $4.5 \%$ for girls and boys, respectively [10]. In this study, the authors did not describe the determinants of overweight and obesity in school-aged children and adolescents. It is important to identify the risk factors that may be predictive of obesity risk in children so that decision-makers can take steps to intervene. The main objectives of the present study were to study a sample of children and adolescents aged 6 to 18 years in the city of Kinshasa in order to examine: 1) characteristics of the eating and activity behaviours of youth by age and gender; 2) the weight status of the youth and related eating and activity behaviours and 3) behavioural factors related to prevalence and determinants of overweight and obesity according to the 2007 World Health Organization (WHO) growth reference for school-aged children and adolescents $[11,12]$.

\section{RESEARCH METHOD}

\subsection{Study design: selection of schools and students}

This study was observational cross-sectional. A multistage sampling method was used to select schools and school-age children. The study was carried out during the period of January and June 2019. The Ministry of National Education provided the authors with a list of all primary and secondary schools active in the city of Kinshasa $(n=996)$. Vocational and special education schools with subjects over 18 years of age and where children did not meet criteria for good health were not included in the study. A total of 568 primary and secondary schools meeting the criteria were grouped according to their location into three strata corresponding to the three existing educational provinces in Kinshasa. Schools were chosen at random alternating selection from primary or secondary until the 18th school was selected. This process resulted in 9 secondary and 9 primary schools chosen to be included in the study.

A power calculation determined that the required sample size for the whole study was 1442 students assuming a prevalence of $8.4 \%$ overweight/obesity ( $8.4 \%$ in sub-Saharan Africa according to the literature [3]. Students were selected by systematic random sampling from each of the 18 schools with blocking on class and sex. Inclusion criteria were that students must be a Congolese national, have the consent of parents or guardians and be between 6 and 18 years old. Students with disabilities and pregnant girls were not included in the study. Students who were randomly selected using the class register were replaced if they did not meet the inclusion criteria or did not consent. A total of 1442 students (774 boys and 668 girls) were enrolled in this study. School record of birth certificate of each enrolled child was used to establish age.

\subsection{Ethical approval}

To conduct this study, ethical approval was obtained from the Ethical Review Committee of Ministry of Health (Ref $\mathrm{N}^{\circ} .143 / \mathrm{CNES} / \mathrm{BN} / \mathrm{PMMF} / 2018$ ). Written Permission was also obtained from the Ministry of Education, which is in charge of primary and secondary schools. Written approval was received from all the respondent parents and school authorities before data collection. Confidentiality was maintained on personal issues by the use of numeric identifiers of subjects.

\subsection{Anthropometry}

It was according to the $\mathrm{WHO}$ protocol that the variables height and weight were measured [13]. For data quality, all measurements have been taken by the principal author. Height in centimeter $(\mathrm{cm})$ and weight in kilogram $(\mathrm{Kg})$ were measured using respectively portable Seca 214 Anthropometer $(\mathrm{Seca} 囚$, Hamburg, Germany) $0.1 \mathrm{~cm}$ near and an OMRON BF 511 body composition scale at $0.1 \mathrm{~kg}$ close. The body mass index (BMI) was obtained using weight in kilograms divided by height in square meters $(\mathrm{kg} / \mathrm{m} 2)$ [12]. WHO AnthroPlus was used to determine height-for-age, weight-for-age and BMI-for-age indices [14] and these indices were expressed as $\mathrm{Z}$ scores. Operational definitions for weight status were as follows $1<-2 \mathrm{SD}$ : 
underweight: thin; <-3 SD "overweight" underweight: very thin; 2) (Z score BMI for age $>+1$ and $\leq+2)$ : overweight; 3) (Z score BMI for age $>+2$ ): obese and 4) (Z score of BMI for age - 2 to +1 ): normal weight. Values were derived from the median values of the 2007 WHO International Growth Benchmark for children aged 5-19 years $[12,14]$. Skin folds in the biceps, triceps, suprailiac, subscapularis and abdominal regions were measured on the right side by a $0.2 \mathrm{~mm}$ Harpenden Gauge [15].

\subsection{Diet and physical activity}

The SPANS 2010 questionnaire [16] was translated into local languages and used to assess the eating behaviour of children during the last 7 days. It included a list of foods and beverages organized by food categories including meat, vegetables, fruit, ice cream and sugary drinks. Respondents were asked to indicate how often they usually consumed each of the listed foods using the following categories: 0 for never or rarely, 1 for 1 to 2 times per week, 2 for 3 to 4 times per week and 3 for more than 5 times per week according to the SPANS protocol. The average score was calculated and classified as low, moderate or high.

The physical activity questionnaire for children (PAQ-C) and adolescents (PAQ-A) [17] was used after translation into local languages. The questionnaire, designed for students aged 6 to 18, contained questions concerning the physical activity of students during the past 7 days. The number of times the student performed any physical activity in the past 7 days was scored. This score was calculated and dichotomized to represent either 'inactive' or 'active'. Demographic information including date of birth, province of origin, municipality, education level of parent, socio-economic status, health status of subjects, and behavior was obtained by parental and student questionnaire and cross-validated using information available in the school registries.

\subsection{Data analysis}

Data were entered in Excel 2010. Characteristics of the eating and activity behaviours of youth by age and gender were conducted by and the associations between weight status of the youth and related eating and activity behaviours were examined by using the comparison tests of proportions (chi-square tests) and the comparison tests of means (test t-student). The behavioural factors related to prevalence and determinants of overweight and obesity was examined by using overweight/obesity as the dependent variable based on the body mass index (BMI) for the age-sex of the children calculated using WHO Anthro plus software V.1.0.4. Covariates included in the models included were socio-demographic factors of children and socio-economic characteristics of their parents [8]. The models also took into account other variables including physical activity, sedentary behaviors and children's eating behaviors. These variables were classified among the behavioral determinants linked to the risk of obesity [6]. SPSS version 21 was used for data analysis. Data for independent variables were presented as proportions in descriptive analysis for the prevalence of overweight/obesity. The search for associations of variables was obtained using the chi-square test and logistic regression. The association between dependent and independent variables was determined by bivariate and multivariate binary logistic regression analyzes.

\section{RESULTS AND DISCUSSION}

\subsection{Results}

Table 1 shows the comparison of socioeconomic status, obesity related behaviors and body fat by age group. Data analysis showed a statistically significant difference between the two age categories for body fat percentage $p<0.001$ and consumption of soft drinks $p<0.05$. Older children fall into the higher consumption of soft drinks as compared to younger children. The majority of older children fall into the high percentage of body fat category as compared to younger children.

Table 2 shows the comparison of SES, obesity related behaviors and body fat by age and sex. Only \% body fat (BF) was significantly different between the younger boys and girls. Younger girls fall into the high $\%$ $\mathrm{BF}$ category as compared to younger boys $(15.7 \pm 7.2$ vs $11.5 \pm 4.0 \mathrm{p}<0.001)$. As for older children, all of the characteristics show significant differences by gender; boys are more likely to report eating a high amount of fruits and vegetables as compared with girls; girls are more likely to report taking more meats and having sweet drinks than boys; boys are more likely to report being more active than girls; and girls had higher BMI than boys $(22.8 \pm 9.8$ vs $12.7 \pm 6.9 \mathrm{p}<0.001)$. Overall, $72 \%$ of the sample was at a healthy weight, $15 \%$ were underweight and nearly $13 \%$ were overweight or obese as shown in Table 3 . The prevalence of overweight and obesity was higher in older students as compared to younger students and in older girls as compared with older boys. Younger age group children were much more likely to be underweight. There were no statistically significant differences in weight status by socio-economic status (SES). 
Table 1. Comparison of SES, obesity related behaviors and body fat by age

\begin{tabular}{|c|c|c|c|}
\hline Variable & $\begin{array}{c}6-9 \text { years } \\
n=413\end{array}$ & $\begin{array}{c}10-18 \text { years } \\
n=1029\end{array}$ & $\mathrm{p}$ \\
\hline SES & & & 0.674 \\
\hline Low & $188(45.5)$ & $495(48.1)$ & \\
\hline Moderate & $188(45.5)$ & $446(43.3)$ & \\
\hline High & $37(9.0)$ & $88(8.6)$ & \\
\hline Eating fruits and vegetables & & & 0.069 \\
\hline Low & $25(6.1)$ & $100(9.7)$ & \\
\hline Moderate & $184(44.6)$ & $442(43.0)$ & \\
\hline High & 204 (49.4) & $487(47.3)$ & \\
\hline Physical activity & & & 0.434 \\
\hline Inactivity & $253(61.3)$ & $653(63.5)$ & \\
\hline Activity & $160(38.7)$ & $376(36.5)$ & \\
\hline Eating meats & & & 0.321 \\
\hline Low & $45(10.9)$ & $95(9.3)$ & \\
\hline Moderate & $192(46.5)$ & $450(43.7)$ & \\
\hline High & $176(42.6)$ & $484(47.0)$ & \\
\hline Having sweet drink & & & 0.021 \\
\hline Low & $100(24.3)$ & $145(14.1)$ & \\
\hline Moderate & $160(38.7)$ & $365(35.5)$ & \\
\hline High & $153(37.0)$ & $519(50.4)$ & \\
\hline$\% \mathrm{BF}$ & & & $<0.001$ \\
\hline Low & $303(73.1)$ & $526(51.1)$ & \\
\hline Moderate high & $90(21.8)$ & $364(35.4)$ & \\
\hline High & $20(4.9)$ & $139(13.5)$ & \\
\hline
\end{tabular}

SES: socio-economic status \%BF: percentage of body fat

Table 2. Comparison of socio-economic status, obesity related behaviors and body fat by age and sex

\begin{tabular}{|c|c|c|c|c|c|c|c|}
\hline \multirow{2}{*}{ Variable } & \multirow{2}{*}{$\begin{array}{c}\text { Total sample } \\
\mathrm{n}=1442\end{array}$} & \multicolumn{2}{|c|}{ 6-9 years } & \multirow[t]{2}{*}{$\mathrm{p}$} & \multicolumn{2}{|c|}{$10-18$ years } & \multirow[t]{2}{*}{$\mathrm{p}$} \\
\hline & & $\operatorname{Girl}(\mathrm{n}=217)$ & Boy $(\mathrm{n}=196)$ & & $\operatorname{Girl}(\mathrm{n}=451)$ & Boy $(\mathrm{n}=578)$ & \\
\hline \multicolumn{8}{|l|}{ SES } \\
\hline Low & $683(47.4)$ & 95 (43.8) & $93(47.4)$ & \multirow[t]{2}{*}{0.593} & $221(49.0)$ & 274 (47.4) & \multirow{3}{*}{0.093} \\
\hline Moderate & $634(44.0)$ & $100(46.1)$ & 88 (44.9) & & 201 (44.6) & 245 (42.4) & \\
\hline High & $125(8.7)$ & $22(10.1)$ & $15(7.7)$ & & $29(6.4)$ & $59(10.2)$ & \\
\hline BMI & $17.2 \pm 2.7$ & $15.9 \pm 2.4$ & $15.6 \pm 2.0$ & 0.196 & $19.7 \pm 3.6$ & $18.4 \pm 3.0$ & 0.000 \\
\hline$\% \mathrm{BF}$ & $16.5 \pm 6.4$ & $15.7 \pm 7.2$ & $11.5 \pm 4.0$ & 0.000 & $22.8 \pm 9.8$ & $12.7 \pm 6.9$ & 0.000 \\
\hline \multicolumn{8}{|c|}{ Eating fruits and vegetables } \\
\hline Low & $125(8.7)$ & $18(8.3)$ & $7(3.6)$ & \multirow{3}{*}{0.126} & 49 (10.9) & $51(8.8)$ & \multirow{3}{*}{0.009} \\
\hline Moderate & $626(43.4)$ & 96 (44.2) & $88(44.9)$ & & $213(47.2)$ & $229(39.6)$ & \\
\hline High & 691 (47.9) & $103(47.5)$ & $101(51.5)$ & & $189(41.9)$ & 298 (51.6) & \\
\hline \multicolumn{8}{|l|}{ Physical activity } \\
\hline Inactive & $906(62.8)$ & $135(62.2)$ & $118(60.2)$ & \multirow[t]{2}{*}{0.676} & $313(69.4)$ & $340(58.8)$ & \multirow[t]{2}{*}{$<0.001$} \\
\hline Active & $536(37.2)$ & $82(37.8)$ & 78 (39.8) & & 138 (30.6) & $238(41.2)$ & \\
\hline \multicolumn{8}{|l|}{ Having sweet drink } \\
\hline Low & $245(17.0)$ & $56(26.8)$ & $44(22.4)$ & \multirow{3}{*}{0.253} & $82(33.4)$ & $63(25.7)$ & \multirow{3}{*}{0.023} \\
\hline Moderate & $525(36.4)$ & 91 (40.9) & $69(35.2)$ & & $190(36.2)$ & $175(33.3)$ & \\
\hline High & 672 (46.6) & $70(32.3)$ & 83 (42.4) & & 191(28.4) & $328(48.8)$ & \\
\hline \multicolumn{8}{|l|}{ Eating meats } \\
\hline Low & $140(9.7)$ & 25 (10.9) & 22 (11.3) & \multirow{3}{*}{0.351} & $51(9.3)$ & $42(7.3)$ & \multirow{3}{*}{0.042} \\
\hline Moderate & $642(44.5)$ & $101(46.5)$ & $91(46.4)$ & & 211 (43.7) & 239 (41.3) & \\
\hline High & $660(45.8)$ & 91 (42.6) & 83 (42.3) & & $189(47.0)$ & 297 (51.4) & \\
\hline
\end{tabular}

SES: socio-economic status \%BF: percentage of body fat

Table 4 shows the characteristics of the sample with overweight and obesity as well as the bivariate and multivariate relationships between determinants examined and overweight/obesity status. Several factors were found to be associated with childhood overweight/obesity in the bivariate analysis as shown in Table 4 including age, sex, gender differences in the older group, $\% \mathrm{BF}$, intake of fruits and vegetables, levels of physical activity and intakes of sweet drinks between the highest and lowest consumers. Multivariate logistic regressions showed that the sex of children, age, \%BF, eating fruits and vegetables, physical activity were significantly associated with childhood overweight/obesity, controlling for other variables. Girls had doubled the risk of having overweight/obesity than boys (aOR=1.9, 95\% CI: 1.2-3.1). Older children were 7 times more likely to have overweight/obesity (aOR $=7.2,95 \% \mathrm{CI}: 3.3-15.7)$ than younger children. Older girls (10 to 18 years old) had doubled the risk of having overweight/obesity than older boys $(\mathrm{aOR}=1.91,95 \% \mathrm{CI}: 1.1-3.2)$. Children with high \% BF were 16 times more likely to have 
overweight/obesity $(\mathrm{aOR}=15.8,95 \% \mathrm{CI}: 7.54-33.24)$ than those who had a moderate or low \% $\mathrm{BF}$. Children who reported having a high consumption of fruits and vegetables decreased the risk of developing overweight and obesity by 0.39 times (aOR $0.39,95 \%$ CI: $0.15-0.97$ ) as compared with those who consumed less. No significant association was observed between levels of physical activity during the week and childhood overweight/obesity. Likewise, no significant association was observed between the consumption of sweet drinks and childhood overweight/obesity in multivariate analysis.

Table 3. Comparison of weight status by age and gender

\begin{tabular}{lcccccc}
\hline Variable & $\mathrm{n}$ & Underweight & Healthy & Overweight & Obese & $\mathrm{p}$ \\
\hline All & 1442 & $219(15.2)$ & $1038(72.0)$ & $131(9.1)$ & $54(3.7)$ & $<0.001$ \\
All age & & & & & & \\
Girls & 668 & $114(17.1)$ & $436(65.9)$ & $85(12.7)$ & $33(4.9)$ & $<0.001$ \\
Boys & 774 & $105(13.3)$ & $602(77.8)$ & $46(5.9)$ & $21(2.7)$ & \\
Age 6-9 & & & & & \\
Girls & $217(52.5)$ & $85(39.2)$ & $127(58.5)$ & $5(2.3)$ & $0(0.0)$ & 0.159 \\
Boys & $196(47.5)$ & $67(34.2)$ & $125(63.8)$ & $2(1.0)$ & $2(1.1)$ & \\
Age 10-18 & & & & & & \\
Girls & $451(43.8)$ & $29(6.4)$ & $409(78.6)$ & $80(17.7)$ & $33(7.3)$ & $<0.001$ \\
Boys & $578(56.2)$ & $38(6.6)$ & $477(82.5)$ & $44(7.6)$ & $19(3.3)$ & \\
SES & & & & & & \\
Low & $683(47.4)$ & $98(44.7)$ & $498(47.4)$ & $59(45.0)$ & $28(51.9)$ & 0.859 \\
Moderate & $634(43.9)$ & $92(42.0)$ & $457(43.7)$ & $62(47.3)$ & $23(42.6)$ & \\
High & $125(8.7)$ & $29(13.2)$ & $83(8.9)$ & $10(7.6)$ & $3(5.6)$ & \\
\hline SES: & & &
\end{tabular}

SES: socio-economic-status

Table 4. Determinants of overweight/obesity (BMI >1SD)

\begin{tabular}{|c|c|c|c|c|c|c|}
\hline \multirow{2}{*}{ Variable } & \multicolumn{2}{|c|}{ Overweight/obesity } & \multicolumn{2}{|c|}{ Univariate analysis } & \multicolumn{2}{|c|}{ Multivariate analysis } \\
\hline & $\mathrm{n}$ & $\mathrm{n}(\%)$ & $\mathrm{p}$ & OR $(95 \% \mathrm{CI})$ & $\mathrm{p}$ & aOR $(95 \% \mathrm{CI})$ \\
\hline \multicolumn{7}{|l|}{ Age } \\
\hline $6-9$ years & 413 & $9(2.2)$ & & 1 & & 1 \\
\hline $10-18$ years & 1029 & $176(17.2)$ & $<0.001$ & $9.26(4.69-18.29)$ & $<0.001$ & $7.20(3.30-15.69)$ \\
\hline \multicolumn{7}{|l|}{ Sex } \\
\hline Boy & 774 & $67(8.7)$ & & 1 & & 1 \\
\hline Girl & 668 & $118(17.7)$ & $<0.001$ & $2.26(1.64-3.12)$ & 0.011 & $1.90(1.16-3.12)$ \\
\hline \multicolumn{7}{|l|}{ Age $6-9$ years } \\
\hline Girl & 217 & $5(2.3)$ & & 1 & & \\
\hline Boy & 196 & $4(2.0)$ & 0.855 & $1.13(0.30-4.28)$ & - & - \\
\hline \multicolumn{7}{|l|}{ Age $10-18$ years } \\
\hline Boys & 451 & $113(25.1)$ & & 1 & & 1 \\
\hline Girls & 578 & $63(10.9)$ & $<0.001$ & $2.73(1.95-3.83)$ & 0.017 & $1.91(1.12-3.23)$ \\
\hline \multicolumn{7}{|l|}{$\% \mathrm{BF}$} \\
\hline Low & 829 & $20(2.4)$ & & 1 & & 1 \\
\hline Moderate & 454 & $54(11.9)$ & $<0.001$ & $5.46(3.22-9.25)$ & $<0.001$ & $6.68(3.52-12.69)$ \\
\hline High & 159 & $111(69.8)$ & $<0.001$ & $9.54(5.53-16.45)$ & $<0.001$ & $15.83(7.54-33.24)$ \\
\hline \multicolumn{7}{|c|}{ Eating fruits and vegetables } \\
\hline Low & 125 & $21(16.8)$ & & 1 & & 1 \\
\hline Moderate & 626 & $92(14.7)$ & 0.008 & $0.73(0.58-0.92)$ & 0.184 & $0.63(0.32-1.25)$ \\
\hline High & 691 & $72(10.4)$ & 0.001 & $0.37(0.31-0.54)$ & 0.042 & $0.39(0.15-0.97)$ \\
\hline \multicolumn{7}{|l|}{ Physical activity } \\
\hline Activity & 536 & $52(9.7)$ & & 1 & & 1 \\
\hline Inactivity & 906 & $133(14.7)$ & 0.007 & $1.60(1.14-2.25)$ & 0.900 & $1.05(0.48-2.29)$ \\
\hline \multicolumn{7}{|l|}{ Eating meats } \\
\hline Low & 140 & $17(8.7)$ & & 1 & & \\
\hline Moderate & 525 & $125(12.6)$ & 0.065 & $1.52(1.01-2.49)$ & & \\
\hline High & 672 & $40(15.7)$ & 0.052 & $1.77(1.03-3.31)$ & & \\
\hline \multicolumn{7}{|l|}{ Having sweet drinks } \\
\hline Low & 245 & $52(16.4)$ & & 1 & & 1 \\
\hline Moderate & 642 & $81(14.1)$ & 0.066 & $1.56(0.96-2.48)$ & 0.612 & $1.24(0.58-2.81)$ \\
\hline High & 660 & $52(10.7)$ & 0.040 & $1.83(1.08-3.29)$ & 0.324 & $1.39(0.72-2.64)$ \\
\hline
\end{tabular}

\subsection{Discussion}

The study found that one in eight children and adolescents aged 6 to 18 years (12.8\%) in Kinshasa schools had either overweight or obesity. This is in agreement with the report by de Onis et al. who predicted that the prevalence of overweight and obesity should reach $12.7 \%$ by 2020 [6]. The prevalence of overweight, obesity and underweight was found to be $9.1 \%, 3.7 \%$, and $15.2 \%$ respectively, in Kinshasa school children enrolled in our study, supporting the conclusion that developing countries face the double burden of obesity and underweight [18, 19]. In detail, girls had a higher prevalence of underweight as 
compared to boys (17.1\% vs. $13.3 \%)$. The relatively high levels of underweight children in school-aged in Kinshasa could be explained by the poor diet, poor eating practices, undernutrition and the high prevalence of infectious and parasitic diseases. In Kinshasa, the younger children were more likely to be underweight as compared to older children, indicating that the younger children need to be under healthcare surveillance for both risks of under and over nutrition. Similar findings were reported by the Ene-Obong and Adebimpe studies in Nigeria [18, 19] and by Pienaar and Jafar respectively in South Africa [20] and Indonesia [21]. In the DRC, this prevalence is slightly increasing as shown by Buhendwa in 2017 [10]. However, other studies conducted in Tanzania and Algeria [22-24] reported a higher prevalence of obesity than the present study. This discrepancy may be attributed to differences related to parent's level of education, physical activity, sedentary lifestyle, economical family status, or even self-perception.

While recognizing the role of nutrition in development of obesity, it is important to note that childhood obesity may result from a complex interaction of genetic, social and environmental factors that may influence eating and physical activity behavior. In terms of nutrition, the majority of children reported a heavy consumption of sweets drinks during the week preceding the survey. In addition, most of the children rarely eat fruits and vegetables and are less active. These types of lifestyle can predispose children to childhood obesity $[25,26]$.

In the present study, girls had higher prevalence of overweight and obesity than boys. These gender differences in weight status have been supported by several studies in the DRC, Nigeria, Tanzania and Zimbabwe [10, 18, 23, 27, 28]. These results are also similar to those reported outside of Sub-Saharan Africa in countries such as India, South Africa, China, Brazil and Vietnam [29]. The gender differences observed in the present and other studies may be attributed to biological or behavioral differences; boys report being be more active than girls resulting in greater energy expenditure. These gender differences may be also caused by socio-economic, social and psychological factors.

In this study, the prevalence of overweight and obesity was higher in older children (10 to 18 years old) than younger children (6 to 9 years old). But there were no variations in weight status by sex in the age group of younger children (6 to 9 years old) than in older children (10-18 years old). Older girls were generally more overweight and obese than their male counterparts. This may be related to the pubescent growth spurt that occurs earlier in females than in males [30, 31]. Under hormonal influence, females experience a faster increase in body size and shape than boys just before puberty [32]. It may also suggest that there are differences in social and psychological factors that boys and girls experience as they grow older, predisposing girls to gain weight while protecting boys from becoming overweight and obese.

In the present study, sex of subjects, age, percentage of body fat and eating fruits and vegetables were significantly associated with childhood overweight/obesity. Previous studies by other authors found similar results [24, 25]. The present study did not find any association between socioeconomic status, physical inactivity and having sweet drinks with overweight/obesity. This is in contrast to studies that have indicated that obesity is associated with physical inactivity, having sweet drinks, SES, urbanization, and ethnicity [33]. Other studies have also shown that when obesity, gender and physical inactivity are evaluated, girls are reported to be more affected than boys [29, 33]. These differences in findings may be related to true differences between populations studied or may reflect bias or measurement error in the measurement tools used to characterize the determinants of overweight and obesity, particularly tools to assess physical activity and eating behaviors.

\section{CONCLUSION}

One in eight participants aged of 6 to 18 years $(12.8 \%)$ in Kinshasa going to primary and secondary schools were either overweight or obese. Gender of respondent, age, percentage of body fat and eating fruits and vegetables were significantly associated with childhood overweight and obesity. The prevalence of overweight, obesity and underweight was reported to be $9.1 \%, 3.7 \%$, and $15.2 \%$ respectively. The DRC must promote school health programs to reduce and halt the increase in the prevalence of overweight and obesity observed in this present study. These programs should result in lifestyle changes for children and adolescents. The governors must encourage the consumption of fruits and vegetables, the limit of sugary drinks, the practice of physical activity. It is desirable that further studies be carried out in rural areas to compare with those in urban areas.

\section{LIMITATIONS AND STRENGTHS}

Notwithstanding the relevance of the present results, the present study has several limitations. Firstly, the sample was drawn from an urban population and may not be representative of the sample obtained in the rural area. Secondly, the study did not assess nor adjust for factors such as birth weight, 
breastfeeding, family type, maternal and childcare, health services, and other environmental factors that could influence the results. Thirdly, as there is no general consensus about BMI cut-points for African children, the cut-points used in the present study, may have overestimated/underestimated the malnourished and over-nutrition prevalence in youth. Fourthly, the results on physical activity and diet should be interpreted with caution since the questionnaires used are limited and may not be valid and reliable in this population. The strengths of this study are numerous. The WHO protocol for anthropometric measurements has been well used. Sample size was 1442 students. Given the socio-economic situation of the DRC, this study is original and can be used for a national study.

\section{ACKNOWLEDGMENTS}

We are extremely grateful to all the respondents who took part in this study and to all the school authorities who helped us with the data collection. Competing Interests: The authors declare no competing interests.

\section{REFERENCES}

[1] Lasserre AM, Chiolero A, Paccaud F., "Bovet P: Worldwide trends in childhood obesity," Swiss Med Wkly 2007, vol. 137, no. 9-10, pp. 157-158, 2007.

[2] $\mathrm{Ng} \mathrm{M}$, Fleming T, Robinson M, et.al., "Global, regional, and national prevalence of overweight and obesity in children and adults during 1980-2013: a systematic analysis for the Global Burden of Disease Study 2013," Lancet, vol. 384, no. 9945, pp. 766-781, 2014.

[3] Muthuri SK, Francis CE, Wachira LJ, Leblanc AG, Sampson M, Onywera VO, Tremblay MS, "Evidence of an overweight/obesity transition among school-aged children and youth in Sub-Saharan Africa: a systematic review," PLoS One, vol. 9, no. 3, p. e92846, 2014.

[4] Negash S, Agyemang C, Matsha TE, Peer N, Erasmus RT, Kengne AP, "Differential prevalence and associations of overweight and obesity by gender and population group among school learners in South Africa: a cross-sectional study," BMC Obes, vol. 4, vol. 29, pp. 1-8, 2017. doi: 10.1186/s40608-017-0165-1

[5] Adom T, De Villiers A, Puoane T, Kengne AP, "Prevalence and correlates of overweight and obesity among school children in an urban district in Ghana," BMC Obes, vol. 6, vol. 14, pp. 1-11, 2019. https://doi.org/10.1186/s40608019-0234-8

[6] de Onis M, Blossner M, Borghi E., "Global prevalence and trends of overweight and obesity among preschool children," Am J Clin Nutr., vol. 92, no. 5, pp. 1257-1264, 2010.

[7] Popkin BM., "The nutrition transition and obesity in the developing world," Journal of Nutrition, vol. 131, no. 3, pp. 871S-873S, 2001. DOI: 10.1093/jn/131.3.871S

[8] Popkin BM, Gordon-Larsen P, "The nutrition transition: worldwide obesity dynamics and their determinants," Int J Obes Relat Metab Disord, vol. 28, Suppl. 3, pp. S2-9, 2004.

[9] Brown T, Moore TH, Hooper L, Gao Y, Zayegh A, Ijaz S, Elwenspoek M, Foxen SC, Magee L, O'Malley C et al: Interventions for preventing obesity in children. Cochrane Database Syst Rev, vol. 7, no. 12, pp. 1-214, 2011.

[10] Buhendwa RA, Roelants M, Thomis M, Nkiama CE, "Nutritional status and height, weight and BMI centiles of school-aged children and adolescents of 6-18-years from Kinshasa (DRC)," Ann Hum Biol, vol. 44, no. 6, pp. 554-561, 2017.

[11] WHO 2007, "Growth reference data for 5-19 years," 2007. [Online]. Available: http://www.who.int/growthref/en/.

[12] de Onis M, Onyango AW, Borghi E, Siyam A, Nishida C, Siekmann J., "Development of a WHO growth reference for school-aged children and adolescents," Bull World Health Organ 2007, vol. 85, no. 9, pp. 660-667, 2007.

[13] WHO:Physical status, "the use and interpretation of anthropometry. Report of a WHO Expert Committee," World Health Organ Tech Rep Ser, vol. 854, pp. 1-452, 1995.

[14] WHO: "AnthroPlus for Personal Computers Manual: Software for Assessing Growth of the World's Children and Adolescents. Bull World Health Organ 2007, Geneva, WHO, 2009. [Online]. Available: http://www.who.int/growthref/tools/en/

[15] Brown N, Scurr J, "The need for a standardised anthropometric protocol for objective assessment of pre- and postoperative breast surgery," Gland Surg, vol. 1, no. 3, pp. 142-145, 2012.

[16] Grunseit AC HL, King L, Rangan A., "A Junk, Food Index for Children and Adolescents," Sydney: Physical Activity Nutrition Obesity Research Group, NSW Ministry of Health; 2013.

[17] Kowalski K CP, Donen R., "The Physical Activity Questionnaire for Older Children (PAQ-C) and Adolescents (PAQ-A) Manual," College of Kinesiology. University of Saskatchewan, 123, 2013.

[18] Ene-Obong H, Ibeanu V, Onuoha N, Ejekwu A, "Prevalence of overweight, obesity, and thinness among urban school-aged children and adolescents in southern Nigeria," Food Nutr Bull, vol. 33, no. 4, pp. 242-250, 2012. 
[19] Adebimpe WO, "Prevalence and knowledge of risk factors of childhood obesity among school-going children in Osogbo, south-western Nigeria," Malawi Med J, vol. 31, no. 1, pp. 19-24, 2019.

[20] Pienaar AE, "Prevalence of overweight and obesity among primary school children in a developing country: NWCHILD longitudinal data of 6-9-yr-old children in South Africa," BMC Obes, vol. 2, no. 2, 2015.

[21] Jafar TH, Qadri Z., Islam M., Hatcher J., Bhutta ZA, Chaturvedi N., "Rise in childhood obesity with persistently high rates of undernutrition among urban school-aged Indo-Asian children," Arch Dis Child, vol. 93, no. 5, pp. 373-378, 2008

[22] Muhihi AJ, Mpembeni RN, Njelekela MA, Anaeli A., Chillo O., Kubhoja S., Lujani B., Maghembe M., Ngarashi D., "Prevalence and determinants of obesity among primary school children in Dar es Salaam, Tanzania," Arch Public Health, vol. 71, no. 1, p. 26, 2013.

[23] Mosha TC, Fungo S., "Prevalence of overweight and obesity among children aged 6-12 years in Dodoma and Kinondoni municipalities, Tanzania," Tanzan J Health Res, vol. 12, no. 1, pp. 6-16, 2010.

[24] Saker M, Merzouk H, Merzouk SA, Ahmed SB, Narce M., "Predictive Factors of Obesity and their Relationships to Dietary Intake in Schoolchildren in Western Algeria," Maedica (Buchar), vol. 6, no. 2, pp. 90-99, 2011.

[25] Muthuri SK, Francis CE, Wachira LJM, LeBlanc AG, Sampson M., Onywera VO, Tremblay MS., "Evidence of an Overweight/Obesity Transition among School-Aged Children and Youth in Sub-Saharan Africa: A Systematic Review," PLoS One, vol. 9, no. 3, 2014.

[26] Adebayo RA, Balogun MO, Adedoyin RA, Obashoro-John OA, Bisiriyu LA, Abiodun OO., "Prevalence and pattern of overweight and obesity in three rural communities in southwest Nigeria," Diabetes Metab Syndr Obes, vol. 7, pp. 153-158, 2014.

[27] Kambondo G., Sartorius B., "Risk Factors for Obesity and Overfat among Primary School Children in Mashonaland West Province, Zimbabwe," Int J Environ Res Public Health, vol. 15, no. 2, p. 249, 2018.

[28] Dos Santos FK, Maia JA, Gomes TN, Daca T., Madeira A., Katzmarzyk PT, Prista A., "Secular trends in growth and nutritional status of Mozambican school-aged children and adolescents," PLoS One, vol. 9, no. 12, pp. 1-15, 2014.

[29] Karki A, Shrestha A, Subedi N., "Prevalence and associated factors of childhood overweight/obesity among primary school children in urban Nepal," BMC Public Health, vol. 19, no. 1, p. 1055, 2018.

[30] Azubuike NC, Onwukwe OS, Onyemelukwe AO, Maduakor UC, Ifeorah IM, Okwuosa CN, Achukwu PU., "Impact of Colocasia esculenta extract and fractions on high-fat diet-induced changes in body weight, adipose tissue and liver of rats, Pak J Pharm Sci, vol. 31, no. 5(Supplementary), pp. 2143-2148, 2018.

[31] Jee YH, Baron J., "The Biology of Stature," J Pediatr, vol. 173, pp. 32-38, 2016.

[32] Mwaniki EW, Makokha AN, "Nutrition status and associated factors among children in public primary schools in Dagoretti, Nairobi, Kenya," Afr Health Sci, vol. 13, no. 1, pp. 39-46, 2013.

[33] Teferi DY, Atomssa GE, Mekonnen TC., "Overweight and Undernutrition in the Cases of School-Going Adolescents in Wolaita Sodo Town, Southern Ethiopia: Cross-Sectional Study Global Nutrition," J Nutr Metab., vol. 2018, no. 11, pp. 1-15, 2018. 